\title{
Inverse Photoemission Spectroscopy of Multiwall Carbon Nanotubes
}

\author{
Samuel Hevia ${ }^{1,2}$, Wladimir Ibáñez ${ }^{1}$, Rodrigo Segura ${ }^{1}$, and Patricio Häberle ${ }^{1}$ \\ ${ }^{1}$ Departamento de Física, Universidad Técnica Federico Santa María, Avenida España 1680, Valparaíso, Chile \\ ${ }^{2}$ Instituto de Física, Universidad Católica de Valparaíso, Av. Brasil 2950, Valparaíso, Chile
}

Received on 04 December, 2005

\begin{abstract}
Multiwall Carbon Nanotubes (MWCNTs) were synthesized by Chemical Vapor Deposition (CVD). Two different procedures were used to grow MWCNT films roughly, aligned in the direction normal to the $\mathrm{SiO}_{2} / \mathrm{Si}(111)$ substrate. Inverse Photoemission Spectroscopy measurements, on these samples, show the existence of resonances which could be traced back to a flat graphene sheet. The unoccupied valence band is fairly similar to that shown by graphite except by an additional intensity in the vicinity of the Fermi level. This resonance could be interpreted both as tubes tips end effects or van Hove singularities in the density of states.
\end{abstract}

Keywords: Multiwall carbon nanotubes; Inverse photoemission

\section{INTRODUCTION}

In the last few years an important number of scientists from different disciplines have concentrated their efforts in the field of nanoscale materials science and technology. In particular, the study of Carbon Nanotubes (CNTs) properties has attracted a special interest. Applications like field emitters, diodes, transistors, flat-panel displays, SPM tips, hydrogen storage, reinforced polymers and molecular delivery are only a few of their possible applications.

Photoemission experiment [1] have demonstrated the occupied electronic structure of MWCNTs arrays is similar to that of highly ordered pyrolytic graphite (HOPG). The main differences are found in the existence of resonances very close to the Fermi edge[1]. In SWCNT these states were attributed to van Hove singularities of the density of states (DOS). Due to the additional quantization along the nanotube circumference, peaks appear symmetrically around the Fermi level $\left(\varepsilon_{F}\right)$ and their energy splitting depends mainly in the diameter of the tubes. In the case of MWCNTs the nature of these additional intensities close to Fermi level is still controversial. They have been attributed to emissions from the tubes tips [2] or to resonances derived from a mixture of the $\pi$ bands and the 1D subband of the nanotubes [3].

It is well known that CNTs can be grown by Chemical Vapor Deposition (CVD) methods, by nucleation of $\mathrm{C}$ around metallic particles of $\mathrm{Fe}, \mathrm{Co}$ and $\mathrm{Ni}$ [4]. Nanoparticles of these metals act as catalyst in the decomposition of hydrocarbonated molecules such as methane, ethane or acetylene.

In this work we used two CVD procedures in order to synthesize aligned MWCNT-films on flat $\mathrm{SiO}_{2} / \mathrm{Si}(111)$ substrates. One of the films was obtained by pyrolysis of Iron (II) Phthalocyanine [5]. This compound has a double function, first supplying iron atoms for the formation of the catalytic nanoparticles and then as a carbon source. In the other method pyrolysis of acetylene over nanostructured Fe films was used [6]. Most of the previous measurements of the electronic structure of these systems have been done on states below $\varepsilon_{F}$ and there is not much information on the empty electronic states. Consequently the aim of this paper is to describe the electronic structure of unoccupied states of MWCNTs, above $\varepsilon_{F}$ using IPS.

\section{EXPERIMENTAL SECTION}

Multiwall Carbon Nanotubes were synthesized by Thermal Chemical Vapor Deposition (CVD) in a horizontal tube furnace ( $4.5 \mathrm{~cm}$ inner diameter).

Growth method I: (sample I) This procedure has been previously reported [5]. In a typical reaction, $0.05 \mathrm{~g}$ of $\mathrm{Fe}-\mathrm{Pc}$ were decomposed over $\mathrm{SiO}_{2} / \mathrm{Si}(111)$ wafers at $1000^{\circ} \mathrm{C}$. The reaction under these conditions takes about $20 \mathrm{~min}$.

Growth method II: (sample II) Previous to the introduction in the furnace, Fe thin films were electron beam evaporated onto $\mathrm{SiO}_{2} / \mathrm{Si}(111)$ [6]. The coverage of the iron films was estimated by Auger Electron Spectroscopy (AES) to be $60 \%$ atomic abundance. The post-treatment annealing $\left(800{ }^{\circ} \mathrm{C} \times 20 \mathrm{~min}\right.$, in the CVD furnace) of this film in the presence of hydrogen, induces the formation of $\mathrm{Fe}$ nanostructures (np-Fe). The CNT growth was carried out by catalytic decomposition of acetylene, at $800{ }^{\circ} \mathrm{C}$, for half an hour.

Scanning Electron Microscopy (SEM) micrographs were obtained from the as prepared samples, in a LEO SEM 1420VP. Transmission Electron Microscopy (TEM) measurements were performed over dispersed samples. The micrographs were taken in a Zeiss EM900 operated at $80 \mathrm{KV}$.

Inverse photoemission spectroscopy (IPS) has been used to obtain information regarding the unoccupied density of states. The signal corresponds to the intensity of photons emitted by a sample bombarded by low energy electrons. In a process much like the generation of X-rays, Vacuum Ultraviolet (VUV) photons are created by an electronic transition in the solid. The intensity of emitted photons is closely dependent on the unoccupied density of states (uDOS). Measurements were performed in a home built isochromat spectrometer [7].

\section{RESULTS}

In both methods, the resulting product include the formation of CNTs densely packed, with a preferential orientation perpendicular to the substrate surface, as can be seen in Figures 1 and 2. Collectively, the CNTs form a thin film of constant thickness, which looks like a turf or carpet. 
Figure 1 shows a series of SEM micrographs of a CNT film grown by pyrolysis of Iron Phthalocyanine (sample I). Figure 1(a), shows the side view of a $18 \mu \mathrm{m}$ thick CNT-film. Figures 1 (b) and 1(c) are a magnification of the lateral profile and the top view, respectively.

Figure 2 shows the SEM micrographs of a CNT array grown by pyrolysis of acetylene over an annealed 60\%-iron film (sample II). The thickness of the CNT-film estimated from figure 2(a) is $82 \mu \mathrm{m}$. Figures 2 (a), 2(b) and 2(c) correspond to the same views shown in Fig. 1.

Transmission electron micrographs of dispersed tubes were also analyzed. For sample I, the mean diameter was $56 \mathrm{~nm}$ whereas for sample II the mean diameter was $49 \mathrm{~nm}$.

From the SEM and TEM micrographs it is possible to verify some differences between samples I and II. The first one is the film thickness, as has been described above. Additionally, sample I presents straight tubes whereas CNTs in sample II have a helical structure.

From the top view it can be verified that although the MWCNT-films show a good microscopic order, most of the tube tips on the top of the film are bended with a random orientation in the plane.

Figure 3 a) shows normal incidence IP spectra of samples I and II taken over a wide energy range. The energies are measured with respect to the Fermi level, value which is fixed using normal incidence IP spectra from an Al sample. The main features in both spectra have been labeled A and A', they are both close to $3[\mathrm{eV}]$ but with a clear shift in energy between both samples, indicating some electronic structure differences between the samples. B' and B are broad resonances located close to an energy of $12,5[\mathrm{eV}]$.
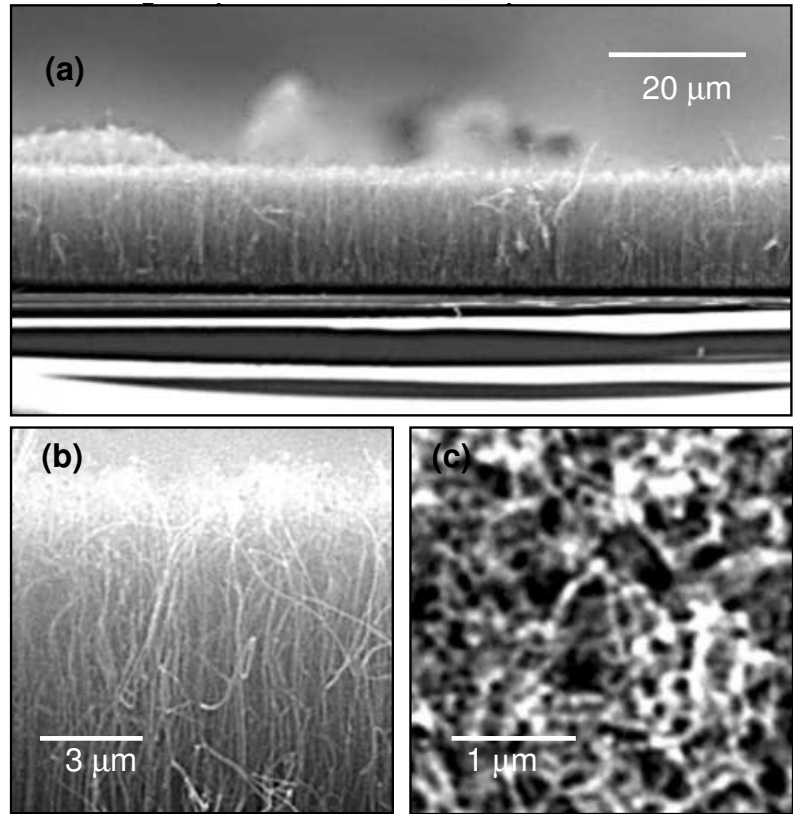

FIG. 1: SEM images of MWCNTs film synthesized by Fe-Pc pyrolysis over a $\mathrm{SiO}_{2}$ substrate. (a) and (b) lateral views; (c) top view.

With the idea of comparing the electronic structure MWCNTs with other systems, in figure 2(b) we present three spec-

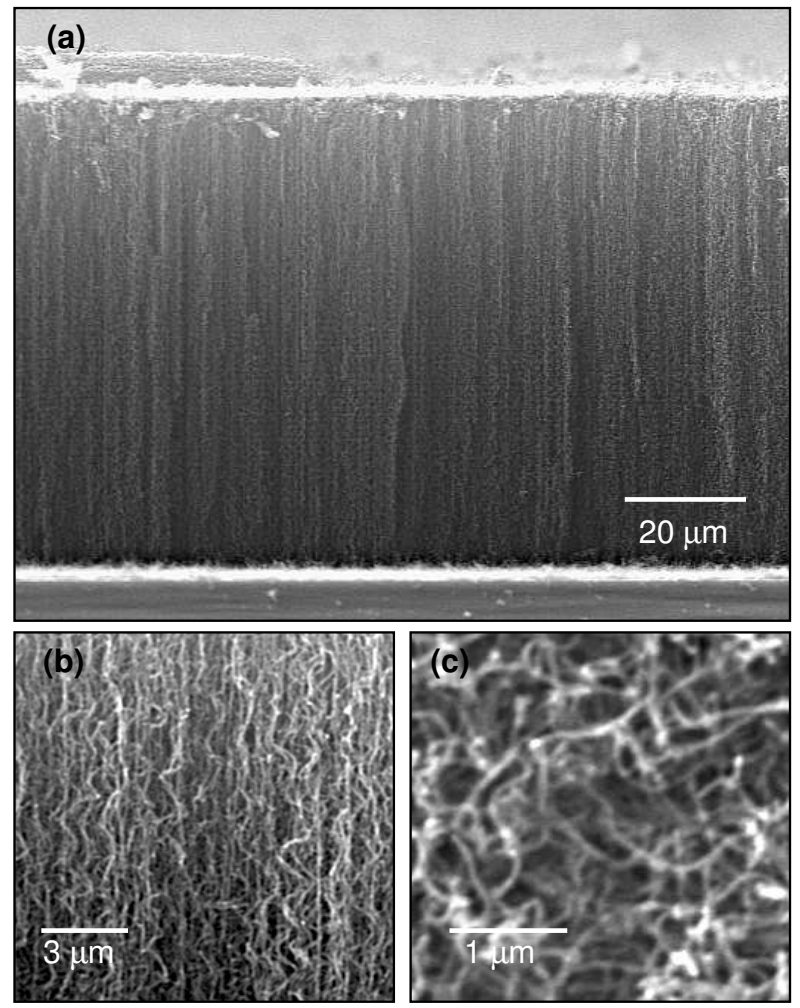

FIG. 2: SEM images of CNTs synthesized from acetylene decomposition over $60 \%-\mathrm{Fe} / \mathrm{SiO}_{2}$. Note the helicoidal symmetry of the tubes. (a) and (b) lateral views; (c) top view.

tra, from the top: a sputtered HOPG sample, MWCNT sample II and data from a fresh HOPG surface. This last sample has two resonances $\mathrm{C}$ and $\mathrm{D}$ in this energy range. Resonance $\mathrm{C}$, at about $1.8 \mathrm{eV}$, has been identified as the $\pi^{*}$ band [8] and D as the $n=1$ surface image state [9] of graphite. The dominant feature in the CNTs sample is resonance A at 3.2 $[\mathrm{eV}]$. An additional, less intense feature ( $\mathrm{C}^{\prime}$ ) is detected at 1.7 $[\mathrm{eV}]$. Sputtered HOPG shows a very broad feature (approximately $2[\mathrm{eV}]$ ) centered around $1.2[\mathrm{eV}]$, raising the IPS intensity closer to $\varepsilon_{F}$ when compared to the fresh HOPG surface.

With the idea of searching for states closer to $\varepsilon_{F}$, we collected a series of spectra with a very low e-beam dose per point to minimize induced damage and thus obtain a higher definition of the spectra in the corresponding energy region. Indeed, as shown in Fig. 2(c) resonance F, close to $0.8[\mathrm{eV}]$, is consistently present in all measured spectra. This resonance is compared with the emission from HOPG, which over the same energy range only shows a smooth increase, with no significant variations of the photon intensity. We have searched, with no success for an angular dependence of the different spectral features. This last result seems to be consistent with the micrographs in Fig. 1, which shows the tubes have no preferential orientation on the top of the CNT film. 


\section{DISCUSSION}

The general belief, at least for large diameter nanotubes, is that most of the electronic structure of Carbon Nanotubes could be traced back to the two-dimensional material from which they are constructed, graphene. This single atomic layer of graphite consists of a 2-D honeycomb structure of $\sigma_{s p 2}$-bonded carbon atoms. The in plane bonding ( $\sigma$ orbitals) form strong covalent bonds with neighboring carbon atoms. Therefore, occupied $\sigma$ and unoccupied $\sigma^{*}$ bonds are formed. The third $\mathrm{C} 2 \mathrm{p}$ electrons are in $2 \mathrm{pz}$-orbitals perpendicular to the plane and form a weaker $\pi$ bond with the 2 pz-orbitals of the atoms in neighboring sites. Due to a weaker bonding, the splitting between the occupied and the unoccupied $\pi$ bands is smaller, thus the $\pi^{*}$ band appears closer to $\varepsilon_{F}$.

Photoemission spectra from CNTs are dominated by emission from $\pi$ and $\sigma$ bands with resonances at $3[\mathrm{eV}]$ and $8[\mathrm{eV}]$ below $\varepsilon_{F}$ respectively [2]. One of the main differences with graphite is the extra emission close to $\varepsilon_{F}$, which has been observed on SWCNTs, MWCNTs and even in more complex carbon "onions" like structures [10]. On SWCNTs, the photoemission data show clear oscillations in the density of states, in agreement with theoretical predictions based of van Hove singularities of the DOS. For MWCNTs this increased intensity has been explained in two ways. Suzuki et al. [2] using photoelectron spectro-microscopy have studied the valence band structure along a single tube axis. Their spectra showed an increased intensity at $\varepsilon_{F}$ only from the spatial region close to the top of the tubes. They explain this behavior as structural defects of the tube tips by the insertions of pentagons on the graphene network, hence implying a higher density of dangling bond at the spherical tips than in the cylindrical side walls. On the other hand Choi et al.[3] using photoemission and density functional calculations postulate the additional $\varepsilon_{F}$ intensity is due to mixing between $\pi$ bands and a truly 1D subband between layers.

If the behavior of the unoccupied valence band in CNTs is similar to the occupied states, as expected, the resulting spectra should resemble those obtained from graphite.

Figure 3(b) shows the IPS spectra from HOPG. Two are the dominant features, one at $1.8[\mathrm{eV}]$ above $\varepsilon_{F}$ which corresponds to the non dispersing $\pi^{*}$ band resonance and the image state at $3.5[\mathrm{eV}]$. For HOPG no increased intensity is observed close to $\varepsilon_{F}$. When we compare the graphite spectra with the one from the MWCNT sample, we can observe a small shoulder in the emission which seems to be related to the $\pi^{*}$ bands, with an obvious reduction in the intensity. Nevertheless this feature is always present in all collected spectra with a small fluctuation in energy as we change to different places in the sample, but it remains unchanged within the resolution of the spectrometer. Above $3.5[\mathrm{eV}]$ we only detect one reproducible structure, in each sample, around 12.5 [eV], (B and B'). By comparing to graphite the energy of this fairly broad resonance corresponds to the $\sigma^{*}$ band. Probably the most significant departure with HOPG is the complete disappearance of the image resonance $\mathrm{D}$, which by the very nature of this state, it requires of a flat surface to exist. If this type of state exists in CNTs the symmetry of the tubes must induce a shift in the
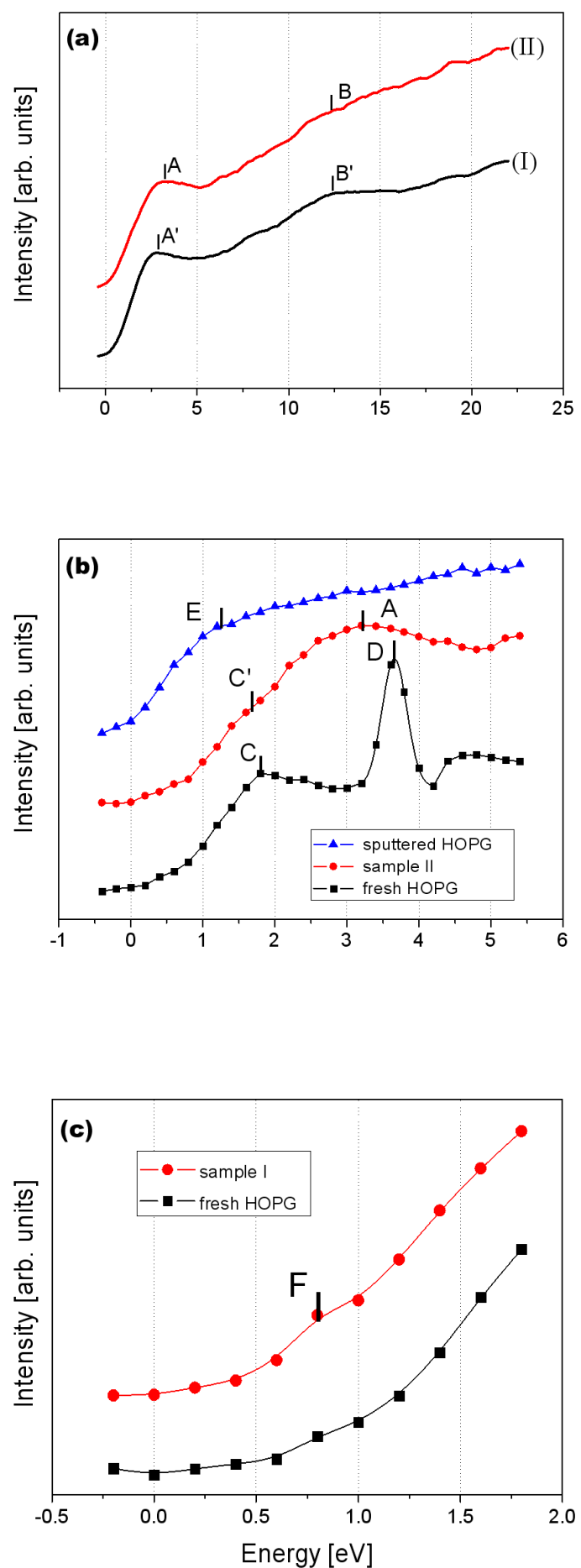

FIG. 3: (a) IPS spectra from samples I y II; energy range: $0.4[\mathrm{eV}]$ to $22[\mathrm{eV}]$. (b) IPS from HOPG, sample II and sputtered HOPG; energy range: $0.4[\mathrm{eV}]$ to $5.4[\mathrm{eV}]$. (c) IPS from sample I and HOPG in the energy range between $0.4[\mathrm{eV}]$ a $2.0[\mathrm{eV}]$. 
energy of the resonance. The idea of collecting spectra from a sputtered HOPG was done to compare the effects of generating many defects on the HOPG surface with the disorder induced states in the NT's spectra. There are two important effects on HOPG: the complete disappearance of resonance $\mathrm{D}$, as expected, and the increased intensity (E) in the IP spectrum close to $\varepsilon_{F}$. These extrinsic surfaces states, which are linked to disorder, have a higher contribution to the DOS for energies of the order of $1[\mathrm{eV}]$ and below. In fact, this result is consistent with the difficulties we had in getting a low noise measurement in CNTs samples close to $\varepsilon_{F}$. Most likely this noise was due to e-beam induced damage of the tubes, hence the need of lowering the dose. Even though both helical and straight tubes are fairly well aligned with the substrate normal, we see no angular dependence of the IPS intensity of any feature. This result together with the SEM images of top of the film led us to believe we only have access to the tips of the tubes or whatever tubes are lying in the top of the CNT film. In both cases no dependence in the orientation of the e-beam is expected. Resonances A and A' could be related to band structure effects of the tubes, but further measurements are required to clarify this point, since the energy of the resonances could be linked to the tube diameter.

For resonance F, which appears robustly in CNT samples, we could not narrow down, to a single one, the justification for the origin of this feature. Two competing explanations are: the resonance is linked to dangling bonds at the tip of the tubes; or the 1D nature of the tubes DOS, which can manifest itself even at these very large tube diameters. This is clearly an open question, which with the information available to us now, can not be resolved.

\section{Acknowledgement}

SH thanks CONICYT for his Doctoral Fellowship. RS thanks MECESUP for his Postdoctoral Fellowship. Partial funding for this research has been provided by Fondecyt, and MSI-Condensed Matter Physics Nucleus (PO2-054-F), Chile.
[1] H. Ishii, H. Kataura et al., Nature 426, 540 (2003).

[2] S. Suzuki, Y. Watanabe, T. Ogino S. Heun et al, Phys. Rev. B 66, 35414 (2002).

[3] J. Choi, S. Lee, Y. Choi, Y. Lee, and J. Jiang, Chem. Phys. Lett 349, 185. (2001).

[4] C. J. Lee, J. Park, Y. Huh, and J. Y. Lee, Chem. Phys. Lett. 343 , 33 (2001); S. Takenaka, M. Ishida, M. Serizawa, E. Tanabe, and K. Otsuka, J. Phys. Chem. B 108, 11464 (2004); M. Paillet, V. Jourdain, P. Poncharal, J. L. Sauvajol, A. Zahab, J. C. Meyer, S. Roth, N. Cordente, C. Amiens, and B. Chaudret, J. Phys. Chem. B 108, 17112 (2004).

[5] R. A. Segura, W. Ibáñez, R. Soto, and P. Häberle, J. Nanosci.
Nanotech. 6, 1945 (2006).

[6] R. A. Segura, M. Flores, P. Häberle, in prep.

[7] P. Häberle, W. Ibañez, R. Esparza, and P. Vargas, Phys. Rev. B63, 235412 (2001)

[8] B. Reihl, J. K Gimzenski, J. M. Nicholls, and E. Tosatti, Phys. Rev. B 33, 5770 (1986).

[9] I. R. Collins, P. T. Andrews, and A. R. Law, Phys. Rev. B 38, 13348 (1988).

[10] Y. U. Butenko, S. Krishnamurti et al, Phys. Rev. B 71, 075420 (2005). 